\title{
Arrested fluid-fluid phase separation in depletion systems: Implications of the characteristic length on gel formation and rheology
}

\author{
J. C. Conrad ${ }^{\text {a) }}$ and H. M. Wyss ${ }^{\text {b) }}$ \\ Department of Physics and SEAS, Harvard University, \\ Cambridge, Massachusetts 02138
}

V. Trappe

Department of Physics, University of Fribourg, CH-1700 Fribourg, Switzerland

S. Manley ${ }^{\text {c) }}$

Department of Physics and SEAS, Harvard University,

Cambridge, Massachusetts 02138

K. Miyazaki ${ }^{\mathrm{d})}$ and L. J. Kaufman

Department of Chemistry, Columbia University, New York, New York 10027

A. B. Schofield

Department of Physics, University of Edinburgh, Edinburgh EH9 3JZ, United Kingdom

D. R. Reichman

Department of Chemistry, Columbia University, New York, New York 10027

$$
\text { D. A. Weitz }{ }^{\mathrm{e})}
$$

Department of Physics and SEAS, Harvard University,

Cambridge, Massachusetts 02138

(Received 17 December 2008; final revision received 2 September 2009; published 12 March 2010)

\section{Synopsis}

We investigate the structural, dynamical, and rheological properties of colloid-polymer mixtures in a volume fraction range of $\phi=0.15-0.35$. Our systems are density-matched, residual charges are

\footnotetext{
${ }^{a)}$ Present address: Department of Chemical and Biomolecular Engineering, University of Houston, Houston, TX 77204.

${ }^{b}$ Present address: Eindhoven University of Technology, ICMS \& WTB, Eindhoven, the Netherlands.

${ }^{c}$ Present address: Institute of Physics of Biological Systems, Swiss Federal Institute of Technology (EPFL), CH-1015 Lausanne, Switzerland.

${ }^{\text {d) }}$ Present address: Institute of Physics, University of Tsukuba, Tsukuba 305-8571, Japan.

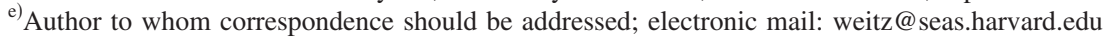


screened, and the polymer-colloid size ratio is $\sim 0.37$. For these systems, the transition to kinetically arrested states, including disconnected clusters and gels, coincides with the fluid-fluid phase separation boundary. Structural investigations reveal that the characteristic length, $L$, of the networks is a strong function of the quench depth: for shallow quenches, $L$ is significantly larger than that obtained for deep quenches. By contrast, $L$ is for a given quench depth almost independent of $\phi$; this indicates that the strand thickness increases with $\phi$. The strand thickness determines the linear rheology: the final relaxation time exhibits a strong dependence on $\phi$, whereas the high frequency modulus does not. We present a simple model based on estimates of the strand breaking time and shear modulus that semiquantitatively describes the observed behavior. (C) 2010 The Society of Rheology. [DOI: 10.1122/1.3314295]

\section{INTRODUCTION}

Colloidal suspensions exhibit a wide range of disordered dynamically arrested states, including repulsive and attractive glasses [Pusey and van Megen (1987); Pham et al. (2002)], ramified gels [Weitz and Oliveria (1984); Lin et al. (1989); Poon et al. (1995); Verhaegh et al. (1997)], and disconnected glassy cluster phases [Sedgwick et al. (2004); Lu et al. (2006)]. The two main control parameters driving this dynamic arrest are the particle volume fraction, $\phi$, and the magnitude of the attractive interaction energy, $U$. In the limit of low $\phi$ and large $U$, irreversible aggregation leads to the formation of fractal gels [Kolb et al. (1983); Meakin (1983); Weitz and Oliveria (1984); Lin et al. (1989)]. In the limit of very low $U$, increasing $\phi$ leads to the arrest in a glassy state, where the particles are permanently trapped within cages of nearest neighbors [Pusey and van Megen (1987)]. Between these two limits, at intermediate $U$ and $\phi$, the interplay between fluid-fluid phase separation and glassy arrest drives the transitions to arrested states [Poon et al. (1995); Verhaegh et al. (1997); Foffi et al. (2005); Manley et al. (2005); Buzzacaro et al. (2007); Cardinaux et al. (2007); Charbonneau and Reichman (2007); Lu et al. (2008)]. This interplay has been recently the subject of intense research, which indicated that parameters such as the range of the attractive potential [Sedgwick et al. (2004); Lu et al. (2006)], the density matching conditions [Shah et al. (2003); Sedgwick et al. (2004)], and the presence of Coulomb interactions [Groenewold and Kegel (2001); Sedgwick et al. (2004); Stradner et al. (2004); Sanchez and Bartlett (2005); Dibble et al. (2006)] can significantly alter the observed behavior. This wide variety of parameters is one of the key difficulties in experimental investigations, where control over all of them is not always easily achieved. To date, the model system offering the best control is the well-studied depletion system composed of poly-methylmethacrylate (PMMA) spheres and polystyrene coils.

In this work we use such a system to explore the phase behavior of colloidal systems with a medium range attraction (polymer-colloid size ratio $\sim 0.37$ ). Our systems are density matched and the effect of charges is minimized by the addition of salt. We explore a volume fraction range of $\phi=0.15-0.35$, where we find that the arrest of the fluid-fluid phase separation leads to both disconnected clusters and space-spanning networks. These networks are composed of interconnected strands, whose characteristic length scale, $L$, decreases as the quench depth increases; however, $L$ is independent of particle volume fraction, implying that the strand thickness increases with $\phi$. The mechanics of these networks are determined by the strand thickness. The high frequency shear modulus depends only weakly on $\phi$, while the relaxation time exhibits a strong dependence. We propose a simple model that accounts for this behavior by incorporating the effects of heterogeneity of the strands, which results in weak points that are most likely to break. This picture also accounts for the origin of the glassy cluster phase through the competition between strand breaking and cluster diffusion. We also rational- 
TABLE I. List of samples with nonzero polymer concentrations studied in this investigation. The polymer concentrations are indicated as polymer concentration in the total sample volume, $c_{p}^{\text {tot }}$, and polymer concentration in the free volume, $c_{p}$, as calculated following Lekkerkerker et al. (1992).

\begin{tabular}{ccc}
\hline \hline & $\begin{array}{c}c_{p}^{\text {tot }} \\
(\mathrm{mg} / \mathrm{mL})\end{array}$ & $\begin{array}{c}c_{p} \\
(\mathrm{mg} / \mathrm{mL})\end{array}$ \\
\hline \multirow{2}{*}{0.15} & 5.88 & 7.93 \\
& 4.75 & 6.41 \\
& 4.50 & 6.98 \\
& 4.27 & 6.62 \\
& 3.74 & 5.80 \\
& 3.54 & 5.49 \\
& 3.28 & 5.09 \\
& 2.94 & 4.56 \\
& 4.04 & 7.43 \\
& 3.72 & 6.84 \\
& 3.35 & 6.16 \\
& 3.03 & 5.57 \\
& 2.87 & 5.28 \\
& 2.63 & 4.84 \\
& 2.29 & 4.21 \\
& 2.59 & 7.64 \\
& 1.95 & 5.75 \\
& 1.74 & 5.13 \\
\hline \hline
\end{tabular}

ize the observed changes in the phase behavior of systems that are not density matched, where shear fluidization due to gravity can interfere with the coarsening process of the spinodal decomposition.

\section{EXPERIMENT}

\section{A. Sample preparation}

The colloidal particles used in this work are PMMA spheres, sterically stabilized by poly-12-hydroxystearic acid [Antl et al. (1986)]; the average colloid radius is $a$ $=136 \mathrm{~nm}$ and the polydispersity $\sim 5 \%$. To minimize sedimentation and scattering, the colloids are suspended in a mixture of cycloheptyl bromide and decahydronapthalene (DHN), which nearly matches both their density, $\rho=1.225 \mathrm{mg} / \mathrm{mL}$, and index of refraction, $n \approx 1.50$. Electrophoresis measurements on similar suspensions indicated that PMMA particles are charged in these solvent mixtures [Yethiraj and van Blaaderen (2003)]. To screen out any long-range Coulombic interactions, we add $\sim 1 \mathrm{mM}$ of an organic salt, tetrabutyl ammonium chloride, to all our samples.

Depletion attractions between the colloids are induced by addition of linear polystyrene (PS) of weight-averaged molecular weight $M_{w}=2 \times 10^{6} \mathrm{~g} / \mathrm{mol}$ and radius of gyration $R_{p}=50 \mathrm{~nm}$, where we parameterize the range of the attraction by $\xi=R_{p} / a \approx 0.37$. The strength of the depletion attraction is set by the concentration of polymer in the free volume, $c_{p}$, which we calculate from the polymer concentration in the total volume, $c_{p}^{\text {tot }}$, via $c_{p}=c_{p}^{\text {tot }}\left(V / V_{\text {free }}\right)$; the free volume is calculated by subtracting both the colloid volume and the volume excluded to the polymer's center of mass from the total volume of the sample [Lekkerkerker et al. (1992)]. The range of polymer concentrations investigated is $c_{p}=4.21-7.93 \mathrm{mg} / \mathrm{mL}$, as reported in Table I; the overlap concentration of the polymer 
is $c_{p}^{*} \approx 8 \mathrm{mg} / \mathrm{mL}$, as determined from viscometry measurements.

To homogenize the samples and break up any particle aggregates, each sample is tumbled for $24 \mathrm{~h}$ prior to each experiment. Both the dynamics and structure of our colloid-polymer mixtures typically evolve for $30 \mathrm{~min}$ after cessation of tumbling or shear applied in a rheometer; we thus equilibrate our samples for at least this time before all experiments.

\section{B. Determination of structural hallmarks}

To determine the spatial arrangement of the colloids in our samples, we use both a scanning microscopy method based on coherent anti-Stokes Raman spectroscopy (CARS) [Zumbusch et al. (1999); Potma et al. (2002)] and static light scattering (SLS). For our CARS experiments, we use two pulsed lasers to generate an anti-Stokes signal at a frequency $\omega_{\mathrm{as}}=2 \omega_{p}-\omega_{s}$ via a nonlinear three-photon optical process, where $\omega_{p}$ and $\omega_{s}$ are, respectively, the frequencies of the pump excitation field and the Stokes excitation field. We tune the lasers to a frequency difference, $\Delta \omega=\omega_{p}-\omega_{s}=2842 \mathrm{~cm}^{-1}$, that excites a Raman-active vibration of the DHN molecules, thereby creating optical contrast between the colloids and the solvent. The resultant CARS signal is proportional to $I_{p}^{2} I_{s}$, where $I_{p}$ is the incident laser intensity and $I_{S}$ is the Stokes laser intensity, and is therefore restricted to contributions from a small focal volume. Consequently the CARS signal is inherently confocal and can be used to acquire images by scanning the sample. Twodimensional images of the samples are obtained by raster scanning the focal point of the lasers over an area of $84 \times 84 \mu \mathrm{m}^{2}$ in $\sim 8 \mathrm{~s}$; the typical time for a $3 \mu \mathrm{m}$ cluster to diffuse its radius in this solvent is $\sim 10 \mathrm{~s}$. The scan speed therefore sets the resolution limit for freely diffusing objects at $\sim 3 \mu \mathrm{m}$; for arrested structures, the resolution limit is $\sim 400 \mathrm{~nm}$.

For our SLS experiments, we use two different set-ups, a simple small angle device and a commercial goniometer, to determine the intensity of the light scattered by our samples, $I(q)$, in two different ranges of wave vectors, $q$. We access a $q$-range of $4.8-34 \mu \mathrm{m}^{-1}$ by using the goniometer equipped with an argon-ion laser operating at a wavelength of $\lambda_{0}=514.5 \mathrm{~nm}$ in vacuo. In this range of wave-vectors, the form factor, $F(q)$, of our particles varies with $q$, for which we must account when determining the static structure factor $S(q)$ from $I(q)$. Because our intensity data are somewhat corrupted by multiple scattering and flare, we adopt the following procedure to determine $S(q)$ : for a given $\phi$, we measure the ensemble-averaged scattered light intensity $I_{\phi}(q)$ for a sample with no added polymer, which we expect to exhibit hard sphere behavior [Pusey and van Megen (1986)]. We then calculate the expected static structure factor $S_{\mathrm{HS}}(q)$ using the Percus-Yevick approximation [Percus and Yevick (1958)] and estimate the "form factor" needed to calculate $S(q)$ as $F_{\phi}(q)=I_{\phi}(q) / S_{\mathrm{HS}}(q)$. The structure factor for our depletion systems is then calculated according to $S(q)=I(q) / F_{\phi}(q)$. For dynamically arrested systems, we obtain the ensemble-averaged intensity by integrating the intensity signal while rotating the sample.

To measure the intensity of scattered light in a lower $q$-range of $0.22-2.9 \mu \mathrm{m}^{-1}$, we illuminate the sample with light from a He-Ne laser $\left(\lambda_{0}=632.8 \mathrm{~nm}\right.$ in vacuo $)$ and image the scattered light onto a screen while allowing the transmitted beam to pass through a hole in the screen. We obtain $I(q)$ by averaging the intensity around rings of constant $q$. In the $q$-range accessible with this setup the particle form factor, $F(q)$, is nearly independent of $q$; thus, we approximate $S(q)$ with $I(q)$. To match the data sets obtained in the low and high $q$-range, we determine the normalization factor that best matches the data sets 
obtained for samples with $c_{p}=0$. For all data sets, we then correct the low $q$ data by this factor.

\section{Determination of dynamical and rheological properties}

To gain insight into the dynamics and rheology of our samples, we perform dynamic light scattering (DLS) and steady and oscillatory shear experiments. We investigate the dynamics at length scales comparable to the particle size $(q a=3.52)$ using DLS. The experiments are performed with the goniometer setup used for the SLS experiments. We quantify the intensity fluctuations by calculating the intensity-intensity correlation function $g_{2}(q, t)=\langle I(q, \tau) I(q, \tau+t)\rangle /\left\langle I(q, \tau)^{2}\right\rangle$ online using a multi-tau correlator (ALV-5000 Multiple Tau Digital Correlator). We collect data for $1800 \mathrm{~s}$ to ensure a good statistical average. For ergodic samples, we use the Siegert relation $g_{2}(q, t)=1+\beta|f(q, t)|^{2}$ to determine the dynamic structure factor $f(q, t)$; the parameter $\beta \approx 1$ depends on the ratio of the speckle size to the collection area of the detector. For non-ergodic samples, we use the Pusey-van Megen method to determine $f(q, t)$ [Pusey and van Megen (1989)]: after each measurement of the time-averaged $g_{2}(q, t)$, we measure the ensemble-averaged scattering intensity $\langle I(0)\rangle_{E}$ by rotating the sample cuvette during a one-minute measurement. We then obtain $f(q, t)$ from a modified Siegert relationship, $f(q, t)=Y-1 / Y+1 / Y\left[g_{2}(q, t)\right.$ $\left.-\sigma^{2}\right]^{1 / 2}$, with $\sigma^{2}=\left\langle I^{2}(0)\right\rangle_{T} /\langle I(0)\rangle_{T}^{2}-1$ and $Y=\langle I(0)\rangle_{E} /\langle I(0)\rangle_{T}$, where $\langle I(0)\rangle_{T}$ is the timeaveraged intensity.

We perform steady-shear-rate and oscillatory measurements at $T \approx 25{ }^{\circ} \mathrm{C}$ using a strain-controlled rheometer (TA-Instruments, ARES). To maximize the measurable range of stress, we use a double-wall couette geometry with a large surface area. Before each measurement, we preshear the sample at a rate of $300 \mathrm{~s}^{-1}$ to break up any structures; subsequently, the sample is allowed to equilibrate for $1200 \mathrm{~s}$ to allow long-range structures to form. During equilibration, we monitor the viscoelastic response of the evolving system in a small-strain oscillatory measurement to ensure that the system reaches a steady state. The viscoelastic response is characterized by performing frequencydependent oscillatory measurements. An oscillatory strain $\gamma_{0}(t)=\gamma_{0} e^{i \omega t}$ is applied to the sample and the resulting time-dependent stress $\sigma(t)=\gamma_{0}(t)\left[G^{\prime}(\omega)+i G^{\prime \prime}(\omega)\right]$ is measured, where the storage modulus $G^{\prime}(\omega)$ and the loss modulus $G^{\prime \prime}(\omega)$, respectively, characterize the elastic and the viscous contributions to the measured stress response. For each sample, we choose the strain amplitude $\gamma_{0}$ to be within the linear viscoelastic regime, which we determine by measuring the strain dependence of $G^{\prime}(\omega)$ and $G^{\prime \prime}(\omega)$ at several frequencies. The flow behavior of our systems is characterized by performing steadyshear measurements as a function of shear rate; the structure and flow are allowed to equilibrate for $15 \mathrm{~s}$ and data are then collected for $15 \mathrm{~s}$ at each shear rate.

To account for the varying contribution of the background solvent to both dynamics and rheology, we determine the concentration dependence of the viscosity $\eta$ of the polymer solutions. We use this data to rescale all times and frequencies by $\eta_{0} / \eta$ and $\eta / \eta_{o}$, respectively, where $\eta_{o}$ is the viscosity of the solvent mixture.

\section{RESULTS AND DISCUSSION}

In Fig. 1, we display the structure factors obtained for our depletion systems in the volume fraction range of $\phi=0.15-0.35$. In the high $q$-range, we observe the same qualitative behavior for all systems: the position of the nearest-neighbor peak is shifted to larger $q a$ as compared to that expected for hard sphere suspensions (solid lines) [Percus and Yevick (1958)]. This shift is nearly independent of polymer concentration, indicating that the nearest-neighbor separation generally decreases as the colloidal particles become 

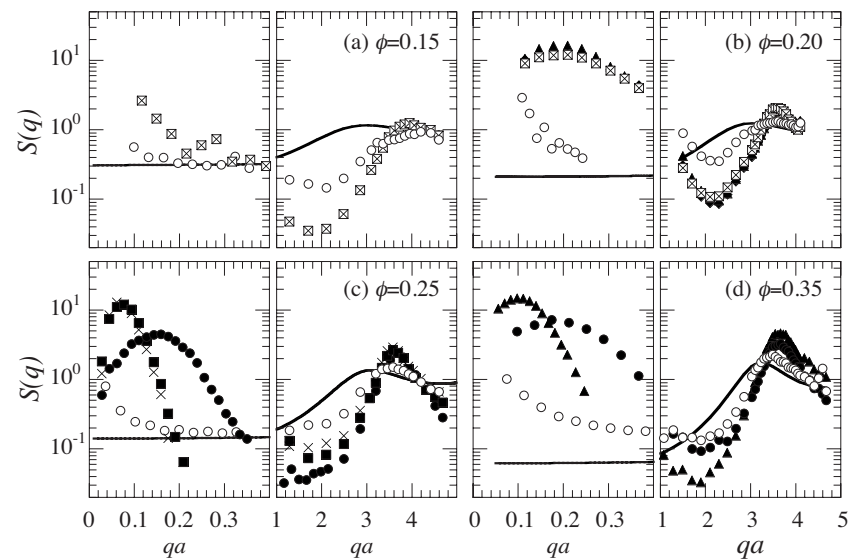

FIG. 1. Wave-vector dependence of the static structure factor obtained in two different $q$-ranges. Solid black lines indicate the structure factor expected for the hard-sphere system as calculated using the Percus-Yevick approximation. (a) $\phi=0.15$ with $c_{p}=6.41 \mathrm{mg} / \mathrm{mL}(\bigcirc), 7.93 \mathrm{mg} / \mathrm{mL}$ ( $\otimes$ ). (b) $\phi=0.20$ with $c_{p}=5.09 \mathrm{mg} / \mathrm{mL}$ $(\bigcirc), 5.49 \mathrm{mg} / \mathrm{mL}(\otimes), 5.80 \mathrm{mg} / \mathrm{mL}(\boldsymbol{\Delta}), 6.62 \mathrm{mg} / \mathrm{mL}(\diamond)$. (c) $\phi=0.25$ with $c_{p}=4.21 \mathrm{mg} / \mathrm{mL}(\bigcirc), 4.84 \mathrm{mg} / \mathrm{mL}$ $(\times), 5.28 \mathrm{mg} / \mathrm{mL}(\boldsymbol{\square}), 7.43 \mathrm{mg} / \mathrm{mL}(\bullet)$. (d) $\phi=0.35$ with $c_{p}=5.13 \mathrm{mg} / \mathrm{mL}(\bigcirc), 5.75 \mathrm{mg} / \mathrm{mL}(\mathbf{\Delta}), 7.64 \mathrm{mg} / \mathrm{mL}$ (๑).

attractive. However, as $c_{p}$ is increased, we detect an additional small shift in the peak position; concurrently the height of the nearest-neighbor peak increases, indicating an increase in the average number of nearest neighbors. This sudden increase in peak height occurs at a critical polymer concentration $c_{p, c}(\phi)$, which depends on $\phi$. We find that $c_{p, c}$ first systematically decreases from $c_{p, c} \sim 7 \mathrm{mg} / \mathrm{mL}$ for $\phi=0.15$ to $c_{p, c} \sim 5.3 \mathrm{mg} / \mathrm{mL}$ for $\phi=0.20$ and $c_{p, c} \sim 4.6 \mathrm{mg} / \mathrm{mL}$ for $\phi=0.25$, and then increases to $c_{p, c} \sim 5.3 \mathrm{mg} / \mathrm{mL}$ between $\phi=0.25$ and $\phi=0.35$. Concomitant with the sudden increase in the height of the nearest-neighbor peak, a second peak appears in the low $q$-range of $S(q)$, indicating the emergence of large length scale structural heterogeneities that are characterized by a well-defined correlation length, $L$.

Additional support for the formation of large length scale structures is obtained from CARS microscopy, as shown for $\phi=0.20$ and $\phi=0.25$ in Fig. 2. Raising the polymer concentration above $c_{p, c}$ for $\phi=0.20$ leads to the formation of large length scale structures, whose characteristic length does not appear to depend on $c_{p}$. This qualitatively agrees with the development of the position of the peak observed in the low $q$-range of $S(q), q_{L}$, which exhibits almost no variation with $c_{p}>c_{p, c}$ for $\phi=0.20$ [Fig. 1(b)]. The agreement between low-angle light scattering and CARS-microscopy data also holds on an absolute scale: the $q$-dependent intensities obtained from the Fourier transform of the CARS images (not shown) exhibit a characteristic turn-over at low $q$ that coincides with the $q_{L}$ obtained in the scattering experiments. To convey this agreement, we report the characteristic length $L=2 \pi / q_{L}$ as circles in Fig. 2. In our series of samples at $\phi=0.20$, we find that the large length scale structures are disconnected for $c_{p}$ just above $c_{p, c}\left(c_{p}\right.$ $=5.49 \mathrm{mg} / \mathrm{mL}$ ); they diffuse slowly during the CARS experiment, exhibiting the typical behavior of a "fluid cluster phase" [Segrè et al. (2001); Lu et al. (2006)]. These fluid clusters coarsen slightly over the duration of our experiment without appearing to coalesce. Increasing the attraction further results in structures that are interconnected and for which we do not observe any temporal evolution. In contrast to the behavior found for $\phi=0.20$, the large length scale structures obtained at $\phi=0.25$ display a striking variation in the characteristic length scale as $c_{p}$ is varied. Samples with a polymer concentration 


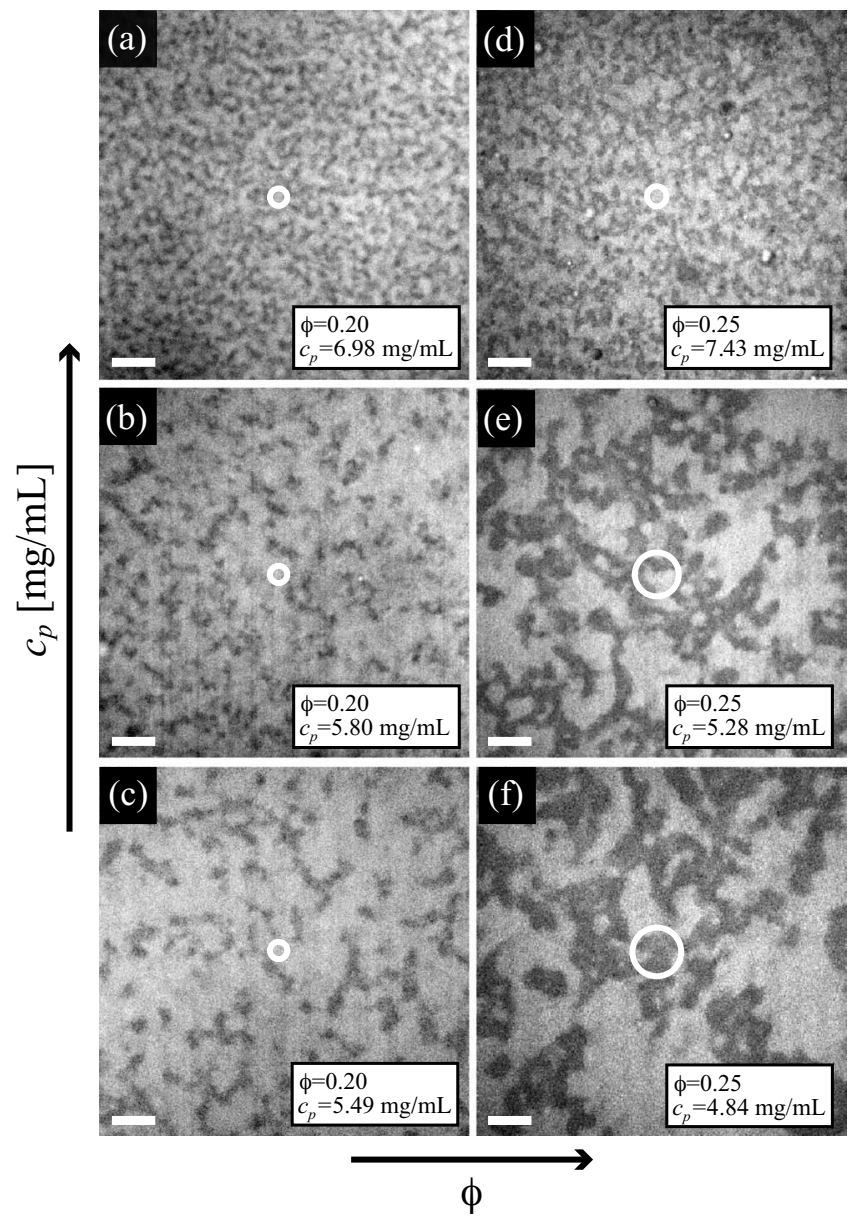

FIG. 2. CARS micrographs obtained at various $c_{p}$ for (a)-(c) $\phi=0.20$ and (d)-(f) $\phi=0.25$. The particles appear dark in these images; the scale bars correspond to $10 \mu \mathrm{m}$. Circles correspond to the characteristic length obtained in small angle light scattering $L \sim 2 \pi / q_{L}$.

just above $c_{p, c}$ exhibit an interconnected structure whose characteristic length is significantly larger than that of samples at larger $c_{p}$. This finding again agrees with the development of the peak position $q_{L}$ [Fig. 1(c)], where we find $L / 2 a=45 \pm 3$ for the samples with a polymer concentration just above $c_{p, c}$, while $L / 2 a=18 \pm 3$ for the samples at larger $c_{p}$. Qualitatively similar behavior is also observed for the samples with $\phi=0.35$ [Fig. 1(d), CARS images not shown]. For the sample with $c_{p}$ just above $c_{p, c}$, coarse network structures with a large characteristic length are formed; for the sample at larger $c_{p}$, the network exhibits a smaller characteristic length. All networks at $\phi=0.25$ and $\phi=0.35$ appear static over the duration of the CARS measurement, which suggests that at this volume fraction arrested gels are obtained for all $c_{p}>c_{p, c^{*}}$.

The sudden appearance of large length scale structures above $c_{p, c}(\phi)$ in conjunction with the sudden increase in particle-particle correlation indicates that $c_{p, c}(\phi)$ corresponds to a well-defined boundary, beyond which the system becomes unstable and starts to phase separate [Verhaegh et al. (1997); Manley et al. (2005); Cardinaux et al. (2007); Lu et al. (2008)]. In this case, the kinetic arrest of the phase separation results from the dynamic arrest of the particles in the colloid-rich regions through an attractive glass 
transition [Manley et al. (2005)]. In this scenario, the variation of $L$ with increasing $c_{p}$ may be understood as a consequence of an arrest in the early stage of phase separation, where the quench depth determines the fastest growing length scale: the deeper the quench, the smaller the length scale [Cahn and Hilliard (1958, 1959)]. However, these length scales are generally predicted to be, at most, of the order of a few particle diameters for colloid-polymer mixtures [Aarts et al. (2004); Bailey et al. (2007)]. By contrast, the characteristic lengths of our arrested systems are rather large, $L / 2 a=15-50$; this suggests that the dynamic arrest occurs during the intermediate stage of phase separation, where the system simultaneously coarsens and densifies in time [Siggia (1979)].

Support for this scenario is obtained by comparing the characteristic lengths obtained in our arrested systems to those obtained during the phase separation of a depletion system similar to the one investigated here. Indeed, Bailey et al. investigated the temporal development of $q_{L}$ after shear melting a PMMA-particle-PS mixture with $a=159 \mathrm{~nm}$, $\xi=0.63, c_{p}^{\text {tot }}=1.285 \mathrm{mg} / \mathrm{mL}$, and $\phi=0.22$ in microgravity [Bailey et al. (2007)]. In this experiment, $q_{L}$ decreases in a short window of $4-80 \mathrm{~s}$ from $\sim 20000$ to $\sim 4000 \mathrm{~cm}^{-1}$, which corresponds to an increase of $L / 2 a$ from $\sim 11$ to $\sim 46$, the range of length-scales characterizing our arrested systems. This indicates that the length scales of our systems are typically obtained in a stage of phase separation where $L$ is already evolving in time. In the experiment performed by Bailey et al., the temporal evolution of $L$ within the time window of interest (4-80 s) indicated that coarsening was still predominantly determined by diffusion. Based on these findings, it therefore seems likely that the kinetic arrest of the phase separation observed in our system occurs during the intermediate stage of phase separation. In this scenario, the variation in the characteristic length scale with increasing $c_{p}$ can be understood as a consequence of an increasing rate of coarsening. As $c_{p}$ is increased, the phase separation is faster and thus less time remains for coarsening to proceed before the arrest condition is reached; this leads to the formation of structures with smaller correlation lengths, in agreement with the observed behavior. Interestingly, we find only little variation of the structural hallmarks as $\phi$ is varied. For sufficiently high $c_{p}$, the network structure is nearly identical for different $\phi$; this can be seen by comparing the structures formed at $\phi=0.20$ and $\phi=0.25$ and $c_{p} \sim 7 \mathrm{mg} / \mathrm{mL}$ [Figs. 2(a) and 2(d)] and the shape and position of the low- $q$ peak in $S(q)$ for $\phi=0.20, \phi=0.25$, and $\phi=0.35$ and $c_{p} \sim 7 \mathrm{mg} / \mathrm{mL}$ [Figs. 1(b) $-1(\mathrm{~d})$ ]. Assuming that the critical density leading to the dynamic arrest of the denser phase is constant at a given $c_{p}, L(\phi) \approx$ const indicates that the density of the denser phase is directly correlated with the characteristic length during the phase separation process.

The origin of the disconnected cluster phase observed at $\phi=0.20$ for $c_{p}$ just above $c_{p, c}$ is not evident. One possibility would be that the disconnected clusters form by a nucleation and growth process, which is arrested when the density of the colloids exceeds the critical density of a glass; here, the glassy clusters are stable if the time it takes to fuse two clusters is much longer than the time it takes for them to diffuse away [Cates et al. (2004); Lu et al. (2006)]. We note, however, that the $q$-dependence of $S(q)$ obtained at $\phi=0.20$ for the connected and disconnected structures are nearly identical; this implies that the kinetic pathways leading to their formation are similar. As will be discussed later, the linear mechanical response function of the connected networks reveals a residual relaxation mechanism, which exhibits a strong dependence on $\phi$. This suggests that the disconnected cluster phases are formed by spinodal decomposition, where the relaxation mechanism is so fast that a connected network cannot be preserved.

To gain further insight into the dynamical arrest conditions of our systems, we probe the dynamics of the individual particles at the nearest-neighbor length scale by determining the dynamic structure factor, $f(q, t)$, at $q a=3.52$. In the absence of polymer $\left(c_{p}=0\right)$, 


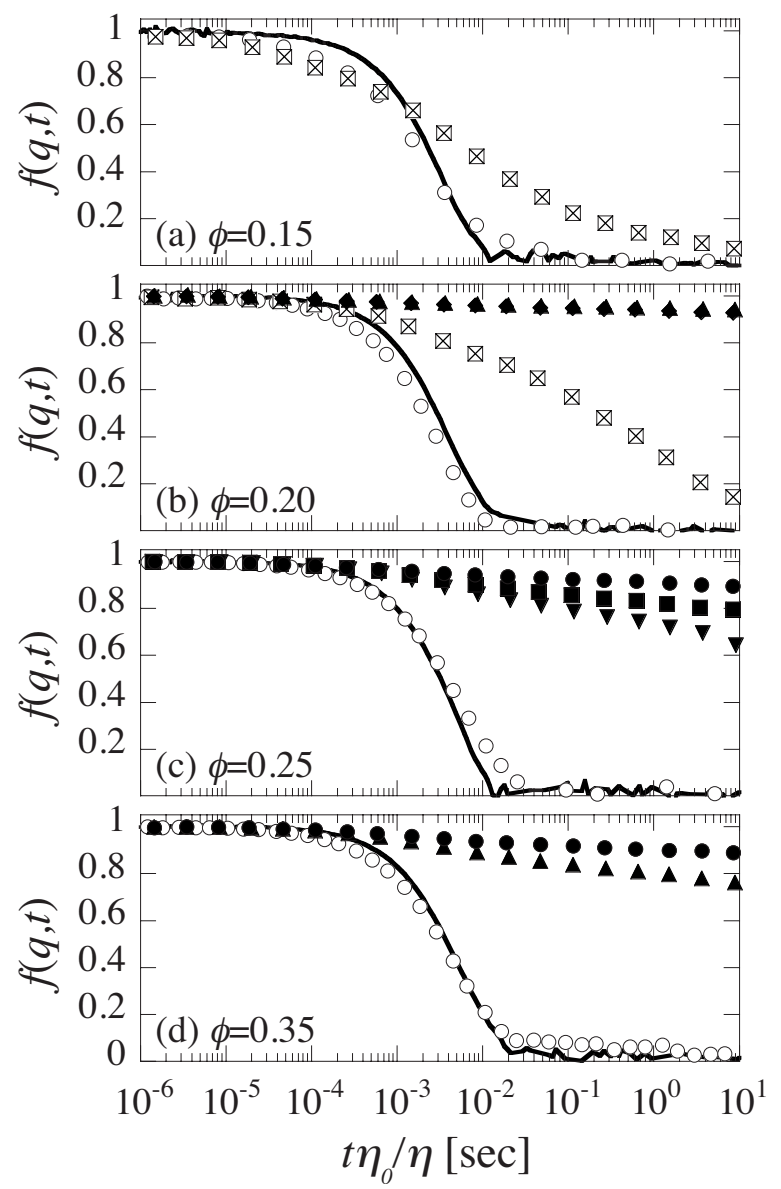

FIG. 3. Dynamic structure factor measured at the peak of the structure factor, $q a \sim 3.52$. To account for the variation in the background viscosity due to the varying polymer concentration, the time axis is rescaled by the ratio of the solvent viscosity to the background viscosity, $\eta_{o} / \eta$. Solid lines denote the behavior of samples with no added polymer $\left(c_{p}=0\right)$. (a) $\phi=0.15$ with $c_{p}=6.41 \mathrm{mg} / \mathrm{mL}(\bigcirc), 7.93 \mathrm{mg} / \mathrm{mL}$ ( $\left.\otimes\right)$. (b) $\phi=0.20$ with $c_{p}$ $=5.09 \mathrm{mg} / \mathrm{mL}(\bigcirc), 5.49 \mathrm{mg} / \mathrm{mL}(\otimes), 5.80 \mathrm{mg} / \mathrm{mL}(\boldsymbol{\Delta}), 6.62 \mathrm{mg} / \mathrm{mL}(\diamond)$. (c) $\phi=0.25$ with $c_{p}=4.21 \mathrm{mg} / \mathrm{mL}$ (○), $4.84 \mathrm{mg} / \mathrm{mL}(\boldsymbol{\nabla}), 5.28 \mathrm{mg} / \mathrm{mL}(\mathbf{\square}), 7.43 \mathrm{mg} / \mathrm{mL}(\bullet)$. (d) $\phi=0.35$ with $c_{p}=5.13 \mathrm{mg} / \mathrm{mL}(\bigcirc), 5.75 \mathrm{mg} / \mathrm{mL}$ (A), $7.64 \mathrm{mg} / \mathrm{mL}(\mathbf{\bullet})$.

$f(q, t)$ decays exponentially for all $\phi$, as shown by the solid lines in Fig. 3, where we report $f(q, t)$ as a function of $t \eta_{o} / \eta$ to account for the changes in the background viscosity due to the presence of the polymer. For $c_{p}<c_{p, c}$, the dynamics of our systems is essentially indistinguishable from that obtained at $c_{p}=0$, indicating that the particles diffuse freely. By contrast, for $c_{p}>c_{p, c}$, the dynamic structure factor exhibits strong deviations from the free-diffusion profile. For $\phi=0.25$ and $\phi=0.35$, the dynamic structure factors decay only partially, exhibiting a nearly time-independent nonzero value at long times for all $c_{p}>c_{p, c}$. This behavior indicates a sharp transition to full arrest of the system in a connected state for all $c_{p}>c_{p, c}$. By contrast, the system at $\phi=0.20$ exhibits a slow structural relaxation for $c_{p}$ just above $c_{p, c}$, in agreement with our observation of diffusing clusters in CARS microscopy. Increasing the polymer concentration further again leads to dynamic arrest at the nearest-neighbor separation, which indicates the existence of an arrested, space-spanning network. For $\phi=0.15$, the dynamics of a sample 


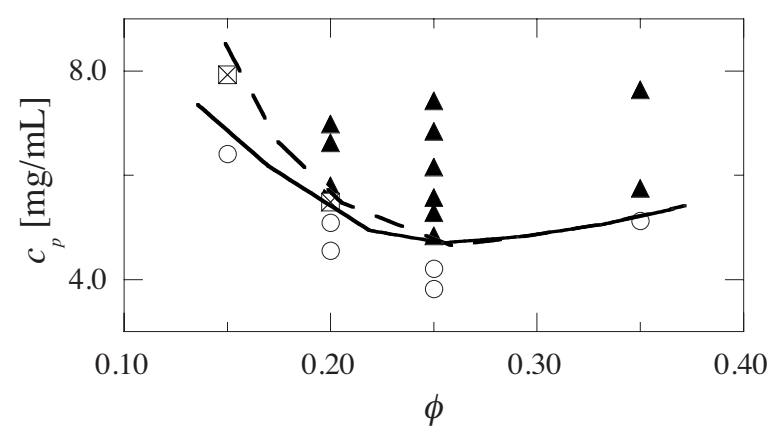

FIG. 4. State diagram of colloid-polymer mixtures with an attraction range of $\xi \sim 0.37$. Symbols indicate the state of the colloidal system: $(\bigcirc)$ dispersed particles; $(\otimes)$ clusters; $(\boldsymbol{\Delta})$ gels. Solid line indicates the boundary between the stable dispersed state and the arrested phase separation states; dashed line indicates the boundary between the fluid clusters and dynamically arrested gel states.

with $c_{p}=7.93 \mathrm{mg} / \mathrm{mL}$ is noticeably slower than free diffusion, even though we observe neither a structure peak at low $q$ nor any large scale structure in CARS microscopy. However, the nearest-neighbor peak is more pronounced for this sample than for the sample with $c_{p}=6.41 \mathrm{mg} / \mathrm{mL}$. We tentatively explain these results with the formation of clusters that are too small to be resolved by CARS and too broadly distributed in size to give rise to a well-developed low $q$ peak.

We summarize the structural and dynamic hallmarks of our system in a phase diagram, shown in Fig. 4. The solid line indicates the boundary beyond which the system undergoes phase separation that becomes arrested when the colloidal-rich regions reach the critical density of a glass. Depending on volume fraction this arrest leads either to disconnected clusters or space spanning networks. The dashed line indicates the boundary beyond which the dynamics of the system becomes arrested, indicating the transition to interconnected space spanning networks.

To gain a better understanding of the various parameters determining the conditions for arrested phase separation, we compare our phase diagram to that reported for other depletion systems. As pointed out in the Introduction, the effects of gravity and charge can significantly modify the phase behavior [Groenewold and Kegel (2001); Shah et al. (2003); Sedgwick et al. (2004); Stradner et al. (2004); Sanchez and Bartlett (2005); Dibble et al. (2006)]. Moreover, the range of the attraction plays a major role in determining both the equilibrium phase behavior and the position and shape of the arrest line [Lekkerkerker et al. (1992); Ilett et al. (1995); Foffi et al. (2002)]. Here we study a buoyancy-matched system, where residual charges are screened by the addition of salt. The range of the attraction, $\xi \sim 0.37$, is intermediate between short and long ranged. Though our measure of the phase behavior is somewhat coarse, our results indicate that arrested phases are formed for all polymer concentrations exceeding $c_{p, c}$. This is at least partly consistent with the phase behavior observed in a depletion system with a shorter range potential, $\xi=0.059$, in which the particle and solvent densities were perfectly matched and all charges were screened by the addition of salt [Lu et al. (2008)]. One of the main findings of that work was that the gelation boundary exactly coincided with the phase separation line. Such coincidence of the gelation boundary with the phase separation line is also observed in our system at $\phi=0.25$ and $\phi=0.35$. By contrast, phase separation that is not interrupted by dynamic arrest of the dense phase can be achieved at even larger $\xi$ as shown for $\xi=0.63$ in [Bailey et al. (2007)]. This dependence of the arrest condition on $\xi$ is consistent with theoretical work on phase behavior [for example, Foffi 
et al. (2002)]: for large $\xi$, the attractive-colloidal-glass-line intersects the fluid-fluid boundary in the decreasing branch of the $T-\phi$ diagram (the increasing branch of the $U-\phi$ diagram), whereas for small $\xi$, the glass-line intersects the fluid-fluid boundary in the top, flat part of the $T-\phi$ diagram (the bottom, flat part of the $U-\phi$ diagram). Thus, for large $\xi$, there is a range of polymer concentrations where phase separation can proceed fully without arrest, whereas for small $\xi$, the fluid-fluid phase separation will invariably be interrupted by vitrification of the denser phase. Our findings indicate that the range of our potential is still sufficiently small to lead to arrested states for all $c_{p}>c_{p, c}$. We note, however, that we did not systematically probe the phase behavior in the direct vicinity of the fluid-fluid phase boundary; we can thus not exclude the possibility that there is a very narrow range of polymer concentrations for which our medium-range potential system would fully phase separate without dynamic arrest.

Interestingly, for systems that are not density matched, full phase separation can also be obtained for short-range attraction. In a PMMA-PS depletion system with $\xi=0.08$, where the density difference between particle and solvent was $\Delta \rho \sim 0.2-0.3 \mathrm{~g} / \mathrm{cm}^{3}$, the hallmarks of classic spinodal decomposition were observed for $c_{p}$ just above $c_{p, c}$, while the formation of transient gels was observed at larger $c_{p}$ [Poon et al. (1995)]. Similarly, for a depletion system with $\xi=0.25$ and an even higher buoyancy mismatch of $\Delta \rho$ $\sim 1.2 \mathrm{~g} / \mathrm{cm}^{3}$, a phase boundary to a two-phase region was observed before crossing the boundary to a transient gel region [Verhaegh et al. (1997)]. Both the formation of transient gels that collapse after a certain latency period and the regime of non-arrested phase separation for $c_{p}$ just above $c_{p, c}$ can be understood as the result of the gravitational load on the system.

As denoted in our data and in recent work on the arrested phase separation of lysozyme [Gibaud and Schurtenberger (2009)], the characteristic length of the arrested state is a function of the quench depth: the shallower the quench, the larger the $L$. This size dependence bears important consequences on the phase behavior of densitymismatched systems. Indeed, for such systems, the capillary length $L_{c}=\sqrt{\gamma /\left(\Delta \rho^{*} g\right)}$ defines the length scale beyond which gravity determines the phase separation behavior [Aarts et al. (2005)]; $\gamma$ and $\Delta \rho^{*}$ are, respectively, the interfacial tension and the density differences between low and high density phases and $g$ is the earth's gravitational acceleration. Depending on density mismatch and quench depth, it is possible that $L_{c}$ is exceeded during phase separation before the arrest conditions are reached. In such cases, the denser phase will never vitrify as shear constantly fluidizes the system; consequently, the phase separation never arrests. For shallow quenches, the characteristic length scale $L$ grows larger before arrest occurs and thus $L_{c}$ is more likely exceeded before arrest in a shallow quench than in a deep quench. Therefore, phase separation proceeds for shallow quenches but becomes arrested for deeper quenches in systems that are not density matched. Accordingly, the gap between the fluid-fluid phase separation boundary and the gel line should depend sensitively on the ratio of $L / L_{c}$, where $L$ is understood here as the length scale determining the potentially arrested state: for $L / L_{c}>1$ phase separation proceeds, whereas for $L / L_{c}<1$ the arrested gel-state is reached. The position of the gel boundary should thus be at $L / L_{c} \sim 1$. In our experiment, where the phase boundary coincides with the arrest boundary, $L_{c}>L$ for all $c_{p}>c_{p, c}$.

Gels formed by arrested spinodal decomposition exhibit features of both colloidal gels and glasses. They form long-range networks and thus exhibit the typical space-spanning features of gels; they dynamically arrest when the local density exceeds a critical threshold and thus also exhibit typical features of glasses. Which of these two characteristics dominates their macroscopic response is not evident. To address this issue, we determine the mechanical behavior of our samples by performing both steady and oscillatory shear 


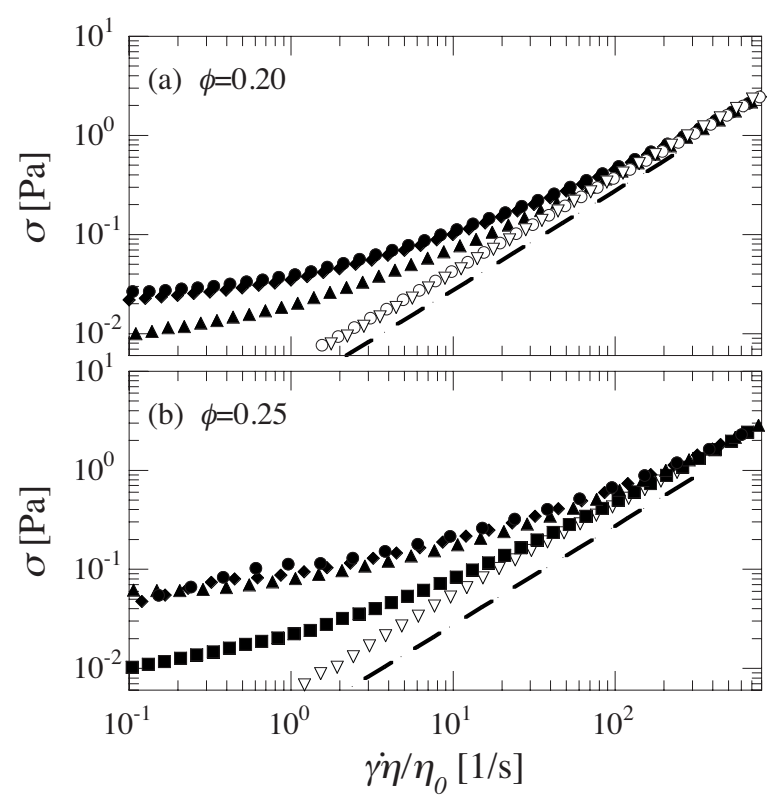

FIG. 5. Stress, $\sigma$, as a function of shear rate $\dot{\gamma}$. To account for the variation in the background viscosity due to the varying polymer concentration, the shear rate axis is rescaled by the ratio of the background viscosity to the solvent viscosity, $\eta / \eta_{o}$. (a) $\phi=0.20$ with $c_{p}=4.56 \mathrm{mg} / \mathrm{mL}(\nabla), 5.09 \mathrm{mg} / \mathrm{mL}(\bigcirc), 5.80 \mathrm{mg} / \mathrm{mL}(\boldsymbol{\Delta}), 6.62$ $\mathrm{mg} / \mathrm{mL}(\bullet), 6.98 \mathrm{mg} / \mathrm{mL}(\bullet)$. (b) $\phi=0.25$ with $c_{p}=4.21 \mathrm{mg} / \mathrm{mL}(\nabla), 5.28 \mathrm{mg} / \mathrm{mL}(\mathbf{\square}), 6.16 \mathrm{mg} / \mathrm{mL}(\boldsymbol{\Delta}), 6.84$ $\mathrm{mg} / \mathrm{mL}(\bullet), 7.43 \mathrm{mg} / \mathrm{mL}(\bullet)$. Dashed lines indicate the viscosity of the background solvent.

measurements. The steady shear experiments essentially confirm the dynamical behavior observed in dynamic light scattering. For samples with $c_{p}<c_{p, c}$, the shear stress increases linearly with the applied shear rate, consistent with Newtonian behavior, as shown in Fig. 5. Deviations from this behavior are observed as the polymer concentration exceeds $c_{p, c}$. For samples with the highest $c_{p, c}$, the stress is independent of shear rate in the low range of shear rates measured, which is a characteristic of solid-like systems exhibiting a dynamical yield stress. This indicates that dynamical and structural arrests are accompanied by the development of mechanical stability. This stress response is remarkably insensitive to polymer concentration when $c_{p}$ is sufficiently large. Moreover, the dynamical yield stress exhibits a surprisingly weak dependence on volume fraction. At the highest polymer concentration investigated, $c_{p} \approx 7 \mathrm{mg} / \mathrm{mL}$, increasing the volume fraction from $\phi=0.20$ to $\phi=0.25$ results in an increase of the yield stress by only a factor of $\sim 2$.

In agreement with these observations, we find for the samples with higher $c_{p}$ that the frequency dependence of the storage and loss modulus exhibits only little variation when $c_{p}$ is varied. As a typical example for the high- $c_{p}$ behavior at $\phi=0.20$ and $\phi=0.25$, we display the frequency dependent response of the two samples with $c_{p} \approx 7 \mathrm{mg} / \mathrm{mL}$ in Fig. 6. Consistent with the expected solid-like properties of the networks, we find that $G^{\prime}$ dominates over $G^{\prime \prime}$ at high frequencies. However, the frequency dependent response also reveals the onset of a dissipative process at low frequencies. The characteristic time of this process exhibits a strong dependence on volume fraction. This is in contrast to the weak increase in the magnitude of the high frequency elastic modulus, which increases by only a factor of $2-4$ as $\phi$ is increased from $\phi=0.20$ to $\phi=0.25$. For the sample with $\phi=0.20$, the relaxation frequency is $\omega_{c} \sim 0.2-0.6 \mathrm{rad} / \mathrm{s}$ near the low end of accessible 


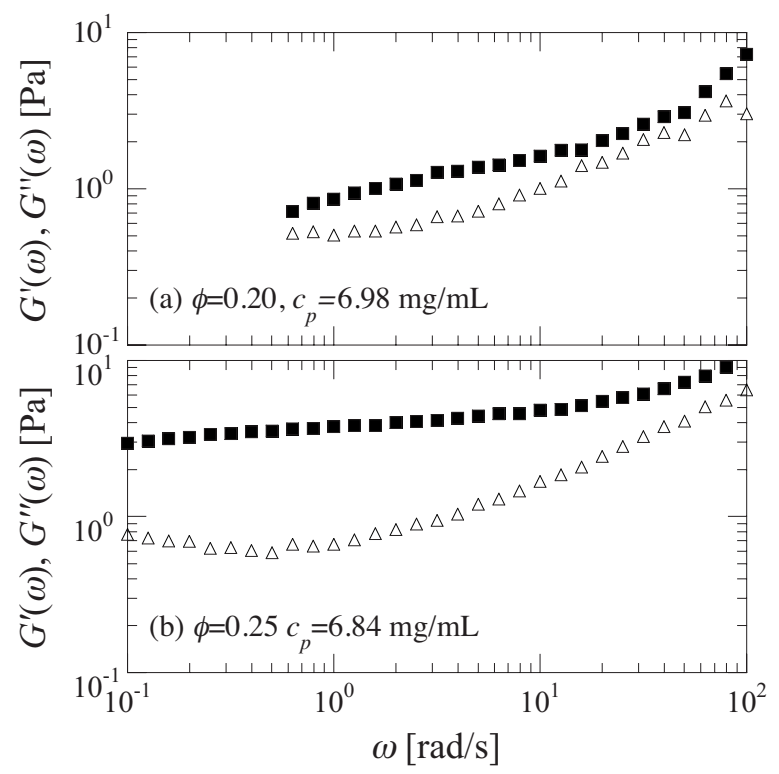

FIG. 6. Frequency dependence of storage $\left[G^{\prime}(\omega), \mathbf{\square}\right]$ and loss $\left[G^{\prime \prime}(\omega), \triangle\right]$ moduli for two samples with comparable values of $c_{p}$ at (a) $\phi=0.20$ and (b) $\phi=0.25$.

frequencies. For the sample with $\phi=0.25$, the relaxation frequency decreases by a factor of 30-100, as estimated by extrapolating the frequency dependence of $G^{\prime}$ and $G^{\prime \prime}$ in the low range of accessible frequencies to $G^{\prime}\left(\omega_{c}\right)=G^{\prime \prime}\left(\omega_{c}\right)$. Although the relaxation time of this process is longer than the time scale accessible in our dynamic light scattering experiments, it is surprising that our DLS data, which probe the dynamics at the particleparticle length scale, show no sign of a final relaxation. This indicates that the dissipative process does not affect the average configuration of the particles.

To account for both the weak $\phi$-dependence of the elastic modulus and the strong $\phi$-dependence of the relaxation time, we examine the structural properties of the two samples under consideration, $\phi=0.20$ and $\phi=0.25$ with $c_{p} \approx 7 \mathrm{mg} / \mathrm{mL}$. Both the SLS data and the CARS microscopy images indicate that the characteristic strand length $L$ is essentially identical for these two samples. As we expect the denser phase to occupy less volume as $\phi$ decreases, a change in $\phi$ must alter the diameter $d_{s}$ of the strands. To estimate the strand diameter, we assume that the local volume fractions of the colloid-rich and colloid-poor phases, $\phi_{1}$ and $\phi_{2}$, do not depend on the total $\phi$ of the sample at a given $c_{p}$; this in turn implies that the fraction of volume occupied by the colloid-rich phase $\chi$ $=\left(\phi-\phi_{2}\right) /\left(\phi_{1}-\phi_{2}\right)$ increases linearly with $\phi$. Since the actual configuration of the network remains unchanged as $\phi$ is varied, $\chi$ should be proportional to the volume of a single strand, $V_{s}=L d_{s}^{2}$; assuming that the number density of strands is $1 / L^{3}$, we can write $\chi=V_{s} / L^{3}=d_{s}^{2} / L^{2}$, such that $d_{s}=L \sqrt{\chi}$.

To estimate the elastic properties of the network, we assume that the dense phase can be described as a isotropic bulk material with an elastic shear modulus given by the interaction energy density, $G_{\text {bulk }} \approx U / a^{3}$, with $U$ the magnitude of the attractive interparticle potential. Additionally, we consider that the load-bearing properties of the network structure are determined by the area fraction of the strands $\left(d_{s} / L\right)^{2}$, which corresponds to $\chi$, and we write $G^{\prime} \approx G_{\text {bulk }} \chi$. Using this expression, we expect for the ratio of the $G^{\prime}$-values at $\phi=0.25$ and $\phi=0.20$ that $G_{\phi=0.25}^{\prime} / G_{\phi=0.20}^{\prime}=\left(0.25-\phi_{2}\right) /\left(0.2-\phi_{2}\right) \approx 1.6$, 
where we estimate $\phi_{2} \sim 0.12$ from the phase diagram in Fig. 4 . This ratio is in fair agreement with our experimental finding: using the $G^{\prime}$-values at the frequency at which $G^{\prime \prime}$ attains its minimum, we obtain a ratio of $\approx 4$, and using the values at a fixed frequency of $10 \mathrm{rad} / \mathrm{s}$, we obtain a ratio of $\approx 2.8$. To further check the validity of this simple description, we evaluate the interaction potential that would best describe the magnitude of the experimental shear modulus according to $U \approx G a^{3} / \chi$, where we estimate from the phase diagram that $\phi_{1} \sim 0.5$. This evaluation yields $U=7 \pm 2 k_{B} T$, in reasonable agreement with predictions of the Asakura-Oosawa theory [Asakura and Oosawa (1954)]. We note, however, that the Asakura-Oosawa theory is only strictly valid at low particle volume fractions, where the depletion effect can be treated as inducing an effective attraction between the colloids. Moreover, recent studies have shown that it is not exact when applied to colloid-polymer mixtures, in particular if partitionings of the polymer between the colloid-rich and the colloid-poor phases are not taken into account [Ramakrishnan et al. (2002)].

Our simple picture of the network morphology should also allow us to estimate the relaxation time of the dissipative process, which we assume to be governed by the breaking of strands. As the strand thickness increases, its cross section contains more interparticle bonds and thus the energy required to break the strand also increases. To estimate the characteristic time scale, we assume a Boltzmann factor with an energy barrier of $U_{\text {eff }}=N_{\text {eff }} U$, where $N_{\text {eff }}$ is the number of bonds that must be broken within the cross section of a strand. We account for the attempt frequency of escaping a potential well with the triangular shape expected for a depletion potential [Shih et al. (1990); Smith et al. (2007); Laurati et al. (2009)]. The relaxation time is then described by

$$
\tau=\frac{\delta^{2}}{D_{s}^{(s)}} \frac{e^{-U_{\mathrm{eff}} / k_{B} T}-\left(1-U_{\mathrm{eff}} / k_{B} T\right)}{\left(U_{\mathrm{eff}} / k_{B} T\right)^{2}},
$$

with $\delta=R_{p}$ the width of the potential well and $D_{s}^{(s)}$ the short time self-diffusion coefficient of the particles within the well, which we estimate from the diffusion coefficient of hard spheres at $\phi=0.5$, where $D_{s}^{(s)} \approx 0.12 D_{0} ; D_{0}$ is the free diffusion coefficient [Segrè et al. (1995)]. For the number of bonds per cross section area of a strand, we presume $\phi_{1} / \pi a^{2}$; accordingly, the number of bonds in the total cross section is given by $N_{\text {eff }}$ $\approx \phi_{1}\left(d_{s} / 2 a\right)^{2}$, such that $N_{\text {eff }} \approx 34$ and $N_{\text {eff }} \approx 55$ for the samples at $\phi=0.20$ and $\phi=0.25$, respectively. Using these values in Eq. (1) yields relaxation times that are many orders of magnitude larger than those observed in the experiments. Moreover, even the ratio between the two time scales, $\tau_{\phi=0.25} / \tau_{\phi=0.20} \approx \exp \left\{\Delta N_{\text {eff }} U / k_{B} T\right\} \approx 10^{64}$, exceeds by many orders of magnitude the experimental finding, where $\tau_{\phi=0.25} / \tau_{\phi=0.20}=30-100$.

To account for this discrepancy, we consider the variation of the strand thickness along its length. As seen in the micrographs of Fig. 2, the strand thickness is indeed not uniform, but shows significant variations. It is reasonable to assume that the strands will break at the weak points where the diameter and thus the number of bonds are minimal. Such a scenario is also suggested by our dynamic light scattering data taken at the nearest-neighbor particle peak, where we find no sign of a final relaxation time. This indicates that on average, the local particle configuration does not change. As we do not observe a temporal evolution of the network structure on the large length scales probed in CARS-microscopy, we can further infer that the local breakage of a strand will not be permanent and that reconnection to the network occurs close to the breaking point. Our macroscopic oscillatory experiment thus captures a very localized relaxation process at the rare breakage point of the network, while our dynamic light scattering experiment probes the average position of the particles, which remains unchanged. To test this inter- 
pretation, we assume that the minimum strand thickness $d_{s(\min )}$ is proportional to the average strand thickness, $d_{s(\min )}=\alpha d_{s}$. We set $\alpha=0.19$ such that the predicted relaxation time for $\phi=0.20, \tau_{\phi=0.2} \approx 10 \mathrm{~s}$, matches the experimentally observed time scale; we then calculate the corresponding relaxation time scale for $\phi=0.25$, where we find $\tau_{\phi=0.25}$ $\approx 840 \mathrm{~s}$ : this relaxation time is well within the range of the experimental estimate $\tau_{\phi=0.25} \approx 300-1000 \mathrm{~s}$. Moreover, the ratio of the two time scales, $\tau_{\phi=0.25} / \tau_{\phi=0.20} \approx 84$, now also agrees with the ratio determined from the experiments, $\tau_{\phi=0.25} / \tau_{\phi=0.20}$ $=10-100$.

While simplistic and semiquantitative, this analysis highlights the significance of the strand thickness on the relaxation time of the material. The dependence of the strand thickness on volume fraction may in fact be at the origin of the fluid cluster phases observed at lower volume fractions. Indeed, the relaxation time of a strand should eventually become so fast that a connected network structure can no longer be maintained. Calculating the relaxation time expected for $\phi=0.15$, we find $\tau_{\phi=0.15} \approx 0.25 \mathrm{~s}$. This time scale is much faster than the time scale for the rotational diffusion of a single strand; using the Broersma relationships for the diffusion of rods [Zero and Pecora (1982)], we estimate a time of $\tau_{\text {rot }} \approx 160 \mathrm{~s}$ for a strand formed at $\phi=0.15$ and $c_{p} \approx 7 \mathrm{mg} / \mathrm{mL}$ to rotate by an angle of $\pi / 4$. This implies that for this system, the vast majority of possible inter-strand bonds are broken on the time scale of diffusion; consequently, the system remains diffusive, in agreement with the observed behavior.

In conclusion, we have investigated the structural, dynamical, and rheological properties of colloidal systems in the volume fraction range $\phi=0.15-0.35$, where a medium range attraction between the colloids is induced by the addition of a non-adsorbing polymer. In this system, the solvent and particle densities are matched and the effect of residual charges is minimized by the addition of salt. Under these conditions, the transition to arrested states coincides with the boundary for phase separation. At lower volume fractions, $\phi \leq 0.20$, disconnected glassy clusters form near the boundary, whereas at higher volume fractions, $\phi \geq 0.25$, space-spanning networks form as soon as the boundary is crossed. Our structural investigations reveal that the characteristic length, $L$, of the networks is a strong function of the quench depth: for shallow quenches, $L$ is significantly larger than that obtained for deep quenches. We suggest that the variation of $L$ with quench depth leads to the significantly different behavior observed for colloid-polymer systems that are not density matched, where phase separation and kinetic arrest occur at different values of $\phi$ [Poon et al. (1995)]. Since for shallow quenches $L$ grows larger before arrest occurs, the likelihood that the capillary length is exceeded before arrest is highest near the phase separation boundary. Once the capillary length is exceeded, the system is shear-fluidized, such that the phase separation process is never arrested; this leads to an off-set of the phase separation boundary and the boundary to kinetically arrested states.

In contrast to the dependence of $L$ on $c_{p}, L$ is almost independent of $\phi$ at a given $c_{p}$; this implies that the strand thickness increases with $\phi$. At higher polymer concentrations, we find that both the dynamic yield stress and the high frequency elastic modulus of the networks depend only weakly on $\phi$. However, the oscillatory shear measurements reveal the existence of a dissipative process with a characteristic time that strongly depends on $\phi$. We present a simple model description that is based on the properties of single strands. This model predicts an exponential dependence of the relaxation time on strand thickness, while the strand thickness enters linearly in the description of the high frequency modulus. The strong dependence of the relaxation time on volume fraction also suggests that a likely origin of the formation of fluid clusters is that the strand breaking-time becomes faster than the strand diffusion-time at sufficiently low volume fractions. By 
describing the elastic and dissipative properties based on the structure of single strands, our simple model description semiquantitatively accounts for the experimentally observed $\phi$-dependence of both the relaxation time and the elastic modulus. It highlights the significance of the strand thickness, thereby providing a good starting point for understanding the relation between structure and rheology of colloidal gels formed by arrested phase separation of systems with medium range attraction.

\section{ACKNOWLEDGMENTS}

The authors gratefully acknowledge fruitful discussions with Irmgard Bischofberger and Peter J. Lu. This work was supported by the NSF (Contract No. DMR-0602684), NASA (Contract No. NAG3-2284), the Harvard MRSEC (Contract No. DMR-0820484), the EPSRC-GB (Contract No. GR/S10377/01) (A.B.S.), and the Swiss National Science Foundation (H.M.W. and V.T.).

\section{References}

Aarts, D. G. A. L., R. P. A. Dullens, and H. N. W. Lekkerkerker, "Interfacial dynamics in demixing systems with ultralow interfacial tension,” New J. Phys. 7(40), doi:10.1088/1367-2630/7/1/040 (2005).

Aarts, D. G. A. L., R. P. A. Dullens, H. N. W. Lekkerkerker, D. Bonn, and R. van Roij, "Interfacial tension and wetting in colloid-polymer mixtures,” J. Chem. Phys. 120, 1973-1980 (2004).

Antl, L., J. W. Goodwin, R. D. Hill, R. H. Ottewill, S. M. Owens, S. Papworth, and J. A. Waters, "The preparation of poly(methyl methacrylate) lattices in nonaqueous media," Colloids Surf. 17, 67-78 (1986).

Asakura, S., and F. Oosawa, "On interaction between two bodies immersed in a solution of macromolecules," J. Chem. Phys. 22, 1255-1256 (1954).

Bailey, A. E., W. C. K. Poon, R. J. Christianson, A. B. Schofield, U. Gasser, V. Prasad, S. Manley, P. N. Segre, L. Cipelletti, W. V. Meyer, M. P. Doherty, S. Sankaran, A. L. Jankovsky, W. L. Shiley, J. P. Bowen, J. C. Eggers, C. Kurta, T. Lorik, P. N. Pusey, and D. A. Weitz, "Spinodal decomposition in a model colloidpolymer mixture in microgravity,” Phys. Rev. Lett. 99, 205701 (2007).

Buzzaccaro, S., R. Rusconi, and R. Piazza, “'Sticky' hard spheres: Equation of state, phase diagram, and metastable gels," Phys. Rev. Lett. 99, 098301 (2007).

Cahn, J. W., and J. E. Hilliard, "Free energy of a nonuniform system. 1: Interfacial free energy," J. Chem. Phys. 28, 258-267 (1958).

Cahn, J. W., and J. E. Hilliard, "Free energy of a nonuniform system. 3: Nucleation in a 2-component incompressible fluid,” J. Chem. Phys. 31, 688-699 (1959).

Cardinaux, F., T. Gibaud, A. Stradner, and P. Schurtenberger, "Interplay between spinodal decomposition and glass formation in proteins exhibiting short-range attractions," Phys. Rev. Lett. 99, 118301 (2007).

Cates, M. E., M. Fuchs, K. Kroy, W. C. K. Poon, and A. M. Puertas, "Theory and simulation of gelation, arrest, and yielding in attracting colloids," J. Phys.: Condens. Matter 16, S4861-S4875 (2004).

Charbonneau, P., and D. R. Reichman, "Systematic characterization of thermodynamic and dynamical phase behavior in systems with short-ranged attraction," Phys. Rev. E 75, 011507 (2007).

Dibble, C. J., M. Kogan, and M. J. Solomon, "Structure and dynamics of colloidal depletion gels: Coincidence of transitions and heterogeneity," Phys. Rev. E 74, 041403 (2006).

Foffi, G., G. D. McCullagh, A. Lawlor, E. Zaccarelli, K. A. Dawson, F. Sciortino, P. Tartaglia, D. Pini, and G. Stell, "Phase equilibria and glass transition in colloidal systems with short-ranged attractive interactions: Application to protein crystallization,” Phys. Rev. E 65, 031407 (2002).

Foffi, G., C. de Michele, F. Sciortino, and P. Tartaglia, "Scaling of dynamics with the range of interaction in short-range attractive colloids," Phys. Rev. Lett. 94, 078301 (2005).

Gibaud, T., and P. Schurtenberger, "A closer look at the arrested spinodal decomposition in protein solutions," J. Phys.: Condens. Matter 21, 322201 (2009). 
Groenewold, J., and W. K. Kegel, “Anomalously large equilibrium clusters of colloids, “J. Phys. Chem. B 105, 11702-11709 (2001).

Ilett, S. M., A. Orrock, W. C. K. Poon, and P. N. Pusey, "Phase-behavior of a model colloid-polymer mixture," Phys. Rev. E 51, 1344-1352 (1995).

Kolb, M., R. Botet, and R. Jullien, "Scaling of kinetically growing clusters," Phys. Rev. Lett. 51, 1123-1126 (1983).

Laurati, M., G. Petekidis, N. Koumakis, F. Cardinaux, A. B. Schofield, J. M. Brader, M. Fuchs, and S. U. Egelhaaf, "Structure, dynamics, and rheology of colloid-polymer mixtures: From liquids to gels," J. Chem. Phys. 130, 134907 (2009).

Lekkerkerker, H. N. W., W. C. K. Poon, P. N. Pusey, A. Stroobants, and P. B. Warren, "Phase behaviour of colloid plus polymer mixtures," Europhys. Lett. 20, 559-564 (1992).

Lin, M. Y., H. M. Lindsay, D. A. Weitz, R. C. Ball, R. Klein, and P. Meakin, "Universality in colloid aggregation," Nature (London) 339, 360-362 (1989).

Lu, P. J., J. C. Conrad, H. M. Wyss, A. B. Schofield, and D. A. Weitz, "Fluids of clusters in attractive colloids," Phys. Rev. Lett. 96, 028306 (2006).

Lu, P. J., E. Zaccarelli, F. Ciulla, A. B. Schofield, F. Sciortino, and D. A. Weitz, "Gelation of particles with short-range attraction," Nature (London) 453, 499-503 (2008).

Manley, S., H. M. Wyss, K. Miyazaki, J. C. Conrad, V. Trappe, L. J. Kaufman, D. R. Reichman, and D. A. Weitz, "Glasslike arrest in spinodal decomposition as a route to colloidal gelation," Phys. Rev. Lett. 95, 238302 (2005).

Meakin, P., "Formation of fractal clusters and networks by irreversible diffusion-limited aggregation," Phys. Rev. Lett. 51, 1119-1122 (1983).

Percus, J. K., and G. J. Yevick, "Analysis of classical statistical mechanics by means of collective coordinates," Phys. Rev. 110, 1-13 (1958).

Pham, K. N., A. M. Puertas, J. Bergenholtz, S. U. Egelhaaf, A. Moussaid, P. N. Pusey, A. B. Schofield, M. E. Cates, M. Fuchs, and W. C. K. Poon, "Multiple glassy states in a simple model system," Science 296, 104-106 (2002).

Poon, W. C. K., A. D. Pirie, and P. N. Pusey, "Gelation in colloid-polymer mixtures," Faraday Discuss. 101, 65-76 (1995).

Potma, E. O., D. J. Jones, J. X. Cheng, X. S. Xie, and J. Ye, "High-sensitivity coherent anti-stokes Raman scattering microscopy with two tightly synchronized picosecond lasers," Opt. Lett. 27, 1168-1170 (2002).

Pusey, P. N., and W. van Megen, "Phase behaviour of concentrated suspensions of nearly hard colloidal spheres," Nature (London) 320, 340-342 (1986).

Pusey, P. N., and W. van Megen, "Observation of a glass-transition in suspensions of spherical colloidal particles," Phys. Rev. Lett. 59, 2083-2086 (1987).

Pusey, P. N., and W. van Megen, "Dynamic light-scattering by non-ergodic media," Physica A 157, 705-741 (1989).

Ramakrishnan, S., M. Fuchs, K. S. Schweizer, and C. F. Zukoski, "Entropy driven phase transitions in colloidpolymer suspensions: Tests of depletion theories," J. Chem. Phys. 116, 2201-2212 (2002).

Sanchez, R., and P. Bartlett, "Equilibrium cluster formation and gelation," J. Phys.: Condens. Matter 17, S3551-S3556 (2005).

Sedgwick, H., S. U. Egelhaaf, and W. C. K. Poon, "Clusters and gels in systems of sticky particles," J. Phys.: Condens. Matter 16, S4913-S4922 (2004).

Segrè, P. N., O. P. Behrend, and P. N. Pusey, "Short-time Brownian motion in colloidal suspensions: Experiment and simulation," Phys. Rev. E 52, 5070-5083 (1995).

Segrè, P. N., V. Prasad, A. B. Schofield, and D. A. Weitz, "Glasslike kinetic arrest at the colloidal gelation transition," Phys. Rev. Lett. 86, 6042-6045 (2001).

Shah, S. A., Y. L. Chen, K. S. Schweizer, and C. F. Zukoski, "Phase behavior and concentration fluctuations in suspensions of hard spheres and nearly ideal polymers," J. Chem. Phys. 118, 3350-3361 (2003).

Shih, W.-H., W. Y. Shih, S.-I. Kim, J. Liu, and I. A. Aksay, "Scaling behavior of the elastic properties of colloidal gels," Phys. Rev. A 42, 4772-4779 (1990).

Siggia, E. D., "Late stages of spinodal decomposition in binary mixtures," Phys. Rev. A 20, 595-605 (1979). 
Smith, P. A., G. Petekidis, S. U. Egelhaaf, and W. C. K. Poon, "Yielding and crystallization of colloidal gels under oscillatory shear," Phys. Rev. E 76, 041402 (2007).

Stradner, A., H. Sedgwick, F. Cardinaux, W. C. K. Poon, S. U. Egelhaaf, and P. Schurtenberger, "Equilibrium cluster formation in concentrated protein solutions and colloids," Nature (London) 432, 492-495 (2004).

Verhaegh, N. A. M., D. Asnaghi, H. N. W. Lekkerkerker, M. Giglio, and L. Cipelletti, "Transient gelation by spinodal decomposition in colloid-polymer mixtures," Physica A 242, 104-118 (1997).

Weitz, D. A., and M. Oliveria, "Fractal structures formed by kinetic aggregation of aqueous gold colloids," Phys. Rev. Lett. 52, 1433-1436 (1984).

Yethiraj, A., and A. van Blaaderen, "A colloidal model system with an interaction tunable from hard sphere to soft and dipolar," Nature (London) 421, 513-517 (2003).

Zero, K. M., and R. Pecora, "Rotational and translational diffusion in semidilute solutions of rigid-rod macromolecules," Macromolecules 15, 87-93 (1982).

Zumbusch, A., G. R. Holtom, and X. S. Xie, "Three-dimensional vibrational imaging by coherent anti-Stokes Raman scattering,” Phys. Rev. Lett. 82, 4142-4145 (1999). 